HIFAN 1527

\title{
Direct Drive Heavy-Ion-Beam Inertial Fusion at High Coupling Efficiency
}

B.G. Logan 1, L.J. Perkins 2, J.J. Barnard 2

1

Lawrence Berkeley National Laboratory

2

Lawrence Livermore National Laboratory

Accelerator Fusion Research Division

Ernest Orlando Lawrence Berkeley National Laboratory

University of California

Berkeley, California 94720

May 2007

This work was supported by the Director, Office of Science, Office of Fusion Energy Sciences, of the U.S.

Department of Energy under Contract No. DE-AC02-05CH11231. 


\title{
Direct drive heavy-ion-beam inertial fusion at high coupling efficiency
}

\author{
B. G. Logan ${ }^{1}$, L. J. Perkins ${ }^{2}$, J. J. Barnard ${ }^{2}$ \\ ${ }^{I}$ Lawrence Berkeley National Laboratory \\ ${ }^{2}$ Lawrence Livermore National Laboratory
}

\begin{abstract}
Issues with coupling efficiency, beam illumination symmetry and Rayleigh Taylor (RT) instability are discussed for spherical heavy-ion-beam-driven targets with and without hohlraums. Efficient coupling of heavy ion beams to compress directdrive inertial fusion targets without hohlraums is found to require ion range increasing several-fold during the drive pulse. Onedimensional implosion calculations using the LASNEX ICF target physics code shows the ion range increasing four-fold during the drive pulse to keep ion energy deposition following closely behind the imploding ablation front, resulting in high coupling efficiencies (shell kinetic energy/incident beam energy of 16 to 18\%). Ways to increase beam ion range while mitigating Rayleigh-Taylor instabilities are discussed for future work.
\end{abstract}

PACS numbers $52.58 \mathrm{Hm}, 52.40 \mathrm{Mj}$ 


\section{Introduction}

In inertial fusion targets, a hollow spherical shell of deuterium-tritium (DT) fuel is imploded by the pressure of a surrounding ablator shell rapidly heated either directly by laser irradiation [1], indirectly by x-rays inside a hohlraum [2-4], or directly by ion beam deposition together with various levels of x-ray assistance [5,7]. Ideally, the most efficient implosions would be done at the peak of rocket efficiency with an ablated mass equal to four times the fuel "payload" mass [8], with a low-Z ablator having small ionization energy, directly with beams to avoid the inefficiency of conversion into x-rays in hohlraums, and with beams that can penetrate the outgoing ablation plasma exhaust and still deposit most of the energy close to the region of peak ablation pressure (ablation front) driving the implosion. Theoretically, the maximum coupling efficiency could be as high as 20 to $30 \%$, depending on the ablator temperature, ionization energy, and profile of beam energy deposition. In comparison, typical laser-driven direct drive coupling efficiency is around $8 \%$, because photons absorb at critical plasma densities much lower than the ablation front [8]), while overall heavy ion hohlraum coupling efficiencies are 2 to $4 \%$, depending on the hohlraum case-to-capsule area ratio, because of $x$-ray conversion and losses to the hohlraum case [3-7]. Symmetry and Rayleigh-Taylor (RT) stability constraints can limit maximum coupling efficiency when the steepness of pressure gradients behind the ablation front are reduced by using off-radial-beam pointing and energy spread [9]. Section II presents results on heavy-ion-beam-driven coupling efficiency from 1-D hydrodynamic implosion calculations using LASNEX [10], when the beam ion range increases several-fold during the implosion. Modest increases $(\sim 25 \%)$ in ion energy during the drive pulse have been used in both light ion [3] and heavy ion [4, 6] indirect-drive hohlraum designs to prevent ion range shortening as the target heated up, but here we consider ion range increasing severalfold during ion direct drive to improve the coupling efficiency. Conclusions are given in Section III, including future 2-D calculations needed to assess RT instability growth with such strongly ramped ion ranges.

Two general problems of heavy ion direct-drive target coupling efficiency that may be improved upon are: (1) decoupling (ion beam deposition which recedes away from the ablation front during the implosion); and (2) beam deposition non-uniformity and RT instability (to be discussed later). Fig. 1 shows two different target examples using spherical ion beam illumination (in general there are cases in between with various amounts of radiation drive [5-7]). In both Fig. 1a and 1b, the arrows depict the radial penetration of ions of assumed constant energy at different times. In the set of arrows labeled "Early in the drive", the ions are allowed to penetrate only a fraction $(<25 \%)$ of the initial ablator thickness to keep the implosion fuel payload on a low adiabat for high compression. If the beam 
ion range were held fixed, the beam energy deposition would migrate radially outward in both target examples, following the outward flow of the ablated mass layer, as depicted by the set of beam arrows labeled "Late in the drive". The growing separation of beam ion energy deposition moving outward from the ablation front moving inward reduces the peak ablation pressure doing the PdV work on the fuel (decoupling).

In spherical targets, decoupling can be mitigated several ways:

(a) the degree of outward flow can be reduced in Fig. $1 b$ if the hohlraum case is thick enough to "tamp" the ablator, but in those cases up to half of the incident beam ion energy can be lost passing through the tamper [5-7];

(b) x-rays can be designed to compensate for the ion decoupling late in the pulse (Fig. 1b) after the outer regions absorbing the beam have been heated to a sufficient temperature $>200 \mathrm{eV}$; however, for the same fuel mass and ion range, the hohlraum contains more mass, thus requiring more beam energy to provide the same implosion velocity;

(c) the ion range can be increased strongly (e.g., by greater than a factor of four when the initial range is $<1 / 4$ of the ablator thickness) to penetrate the ablated plasma late in the drive pulse;

(d) the ion range at constant ion energy can also be increased strongly by heating the plasma electrons to thermal velocities exceeding the ion beam speed, as will be discussed in the next section.

In one early study [11], the ion energy and range was increased several fold in the second of two sequential drive pulses, but for a different purpose to "explode the pusher" (remaining ion absorber) after the target had imploded to a small fraction of the initial radius (requiring much smaller beam spots and higher beam intensities in the second pulse). By contrast, in this present paper we consider employing a strongly increasing ion range to allow the beam to follow the ablation front inward during the implosion to improve coupling efficiency. Using a timeincreasing (ramped) proton beam voltage from 5 to $40 \mathrm{MV}$ during the drive pulse, Havazelet, et. al. [12] calculated total beam input energy decreased from 6.6 MJ (for gain 35 with constant voltage) to $4.1 \mathrm{MJ}$ (for gain 53 with ramped voltage) for relatively lower gain magnetized targets. Recent experiments on the Neutralized Drift Compression Experiment (NDCX) at LBNL have successfully used ramped acceleration voltages to longitudinally compress heavy ion beams by more than a factor of 60 [13]; we note here that this technique might be applied to direct-drive fusion targets, as well as to future NDCX experiments to study hydrodynamic coupling efficiency in planar targets with variations in the amount of energy ramping of the ion beam. 


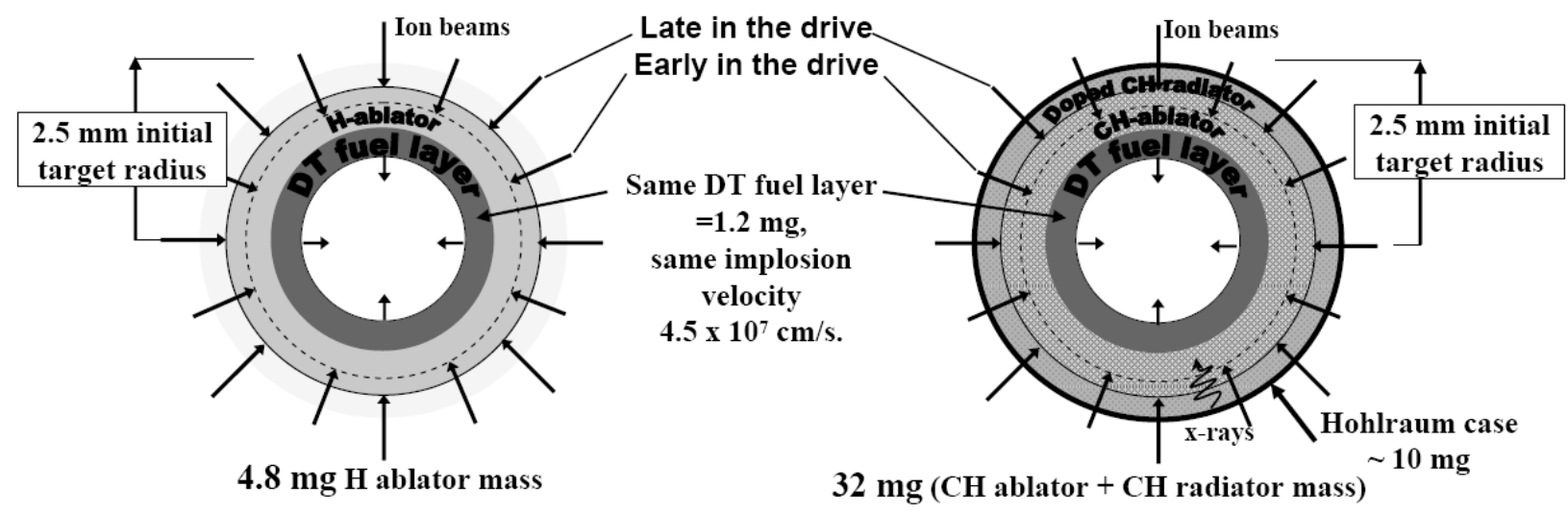

(a) Ablative Direct Drive Example $\rightarrow 1$ MJ drive @ gain 50 (1-D Lasnex)

\section{(b) Spherical-Illumination Indirect Drive Example $\rightarrow 2.6 \mathrm{MJ}$ drive@ gain 19, (model estimate)}

FIG. 1. Examples for ion-driven ablative direct drive (1a) and close-coupled spherical hohlraums (1b).

\section{LASNEX 1-D Implosion Calculations in the ion-beam-driven ablative rocket regime}

The dominant contribution to heavy ion energy loss at the ion energies we are considering is fast ion drag on slower target electrons. Seeking a higher coupling efficiency, we performed 1-D LASNEX implosion calculations for the ablative-drive regime like Fig. 1a using $50 \mathrm{MeV}$ Argon ion beams (constant incident ion energy) whose speed would fall below the electron thermal velocity as the beam power heated the ablator electron temperature above $460 \mathrm{eV}$, which lengthens the ion range during the drive. The LASNEX code [10] we used includes a classical formulation of ion energy loss to calculate the radial profiles of ion beam energy deposition $\mathrm{dE} / \mathrm{dx}$ (energy deposited per unit distance). The model is essentially given by [14]:

$$
\left.\left.-\frac{d E}{d x}=\left[\frac{4 \pi e^{2}}{m_{e} c^{2}}\right] \frac{N_{0} \rho_{T}}{A_{T}}\right] \frac{Z_{e f f}^{2}}{\beta^{2}}\right]\left\{\left(Z_{T}-\bar{Z}\right)\left(\log \Lambda_{B}+R\right)+\bar{Z} G\left(\beta / \beta_{e}\right)\left(\log \Lambda_{F}+R / 2\right)\right\}
$$

Here $\rho_{\mathrm{T}}, A_{\mathrm{T}}, Z_{\mathrm{T}}$, and $\bar{Z}$ are the target density, target atomic weight, target atomic number and target ionization state $\Lambda_{B}=2 m_{e} c^{2} \beta^{2} / \bar{I} ; \Lambda_{F}=m_{e} c^{2} \beta^{2} /\left(\hbar \omega_{p}\right) ;$ and $\mathrm{G}(\mathrm{x})$ is the Chandrasekhar function $=\operatorname{erf}(\mathrm{x})-\mathrm{x} \mathrm{d}(\operatorname{erf}(\mathrm{x})) / \mathrm{dx} \approx 1$ for $\mathrm{x}$ $>>1$, where $\operatorname{erf}(\mathrm{x})$ is the error function of $\mathrm{x} ; \bar{I}$ is the average ionization potential, given approximately by Bloch's rule as $\bar{I} \approx 0.01 Z_{T} \mathrm{keV} ; \omega_{p} \equiv \sqrt{4 \pi e^{2} n_{e} / m_{e}}=56416 \sqrt{n_{e}} \mathrm{~s}^{-1}$ is the plasma frequency, $n_{e}=\bar{Z} N_{0} \rho_{T} / A_{T}$ and $\hbar \omega_{p} \approx 3.7 \times 10^{-14} \sqrt{n_{e}} \mathrm{keV}$, where $\mathrm{n}_{\mathrm{e}}$ is measured in $\mathrm{cm}^{-3}$. Also, $\beta=v / c$ is the ion beam velocity in units of the 
speed of light $c$, and the Lorentz factor of the ion beam $\gamma$, is given by $\gamma=1 / \sqrt{1-\beta^{2}}=1+E / M c^{2}$, where $E$ is the kinetic energy of the ion beam, $\mathrm{Mc}^{2}$ is the rest energy of an ion with atomic mass $\mathrm{A}_{\text {IonBeam.. The electron rest energy }}$ is $m_{e} c^{2}$. $R$ is the relativistic correction, given by $R=2(\log \gamma)-\beta^{2}$. Also, the Betz formula for the effective charge state of the ion was used: $Z_{\text {eff }}=Z_{\text {IonBeam }}\left[1-\exp \left(-137 \beta_{\text {eff }} / Z_{\text {IonBeam }}^{0.69}\right)\right]$ where $\beta_{\text {eff }}^{2}=\beta^{2}+\beta_{e}^{2}$ and with $\gamma_{e}=1 / \sqrt{1-\beta_{e}^{2}}=1+k T_{e} / m_{e} c^{2}$.

The model uses the classical Bethe-Bloch formulation for stopping [14], including terms for both bound and free electrons and is sufficiently accurate for cases considered here in which (a) low-Z ablators are hot enough to be fully ionized or nearly-fully ionized, (b) the dominant mechanism for beam ion energy loss is by the collective drag of many plasma electrons within a Debye shielding distance, and (c) there are sufficient free electrons that are slower than the ion speed over most of the ion range, and (d) the projectile ion energy and mass are moderate enough to neglect nuclear fragmentation.

Figure 2 below shows the target construction (2a), and pulse shape used (2b) for a LASNEX calculation which we have analyzed most. The ablator is solid DT wicked into low density $\left(100 \mathrm{mg} / \mathrm{cm}^{3}\right) \mathrm{CH}$ :DT filled foam; the ablator has an atomic ratio of $(\mathrm{CH})_{1}(\mathrm{DT})_{6}$ with a $43 \%$ carbon mass fraction. This fuel payload of $1.2 \mathrm{mg}$ is $31 \%$ of the initial total initial target mass implosion velocity (where the peak of the rocket efficiency would be at a fuel payload fraction of $20 \%$ ) and where the final implosion velocity is $V_{\text {exh }} * \ln \left(m_{\text {initial }} / m_{\text {final }}\right) \sim 4.45 \times 10^{7} \mathrm{~cm} / \mathrm{s}$ (not optimized). Nonetheless, this un-optimized case still gave an overall beam-to-ignition coupling efficiency (fuel payload energy $) /($ total beam input $)=0.159 \mathrm{MJ} / 1 \mathrm{MJ}=15.9 \%$. Note that in some of the early references $[5,6]$, metallic pushers were included with the DT fuel in defining a coupling efficiency to the "payload" including the pusher; here, beam-to-DT-fuel coupling refers to energy given only to the $1.2 \mathrm{mg}$ of fuel.

It is interesting to note that the characteristics of heavy-ion direct drive - i.e., high ablation rates, lower ablation velocities - are analogous to those of indirect drive with x-rays, whereas conventional laser direct drive exhibits low ablation rates and high exhaust velocities, requiring the latter to operate at significantly higher fuel payload fractions removed from the peak of the rocket efficiency curve. For these reasons, we also expect that heavy-ion direct drive will provide similar beneficial ablation-velocity-stabilization of high-mode Rayleigh-Taylor instabilities seen with indirect x-ray drive. 

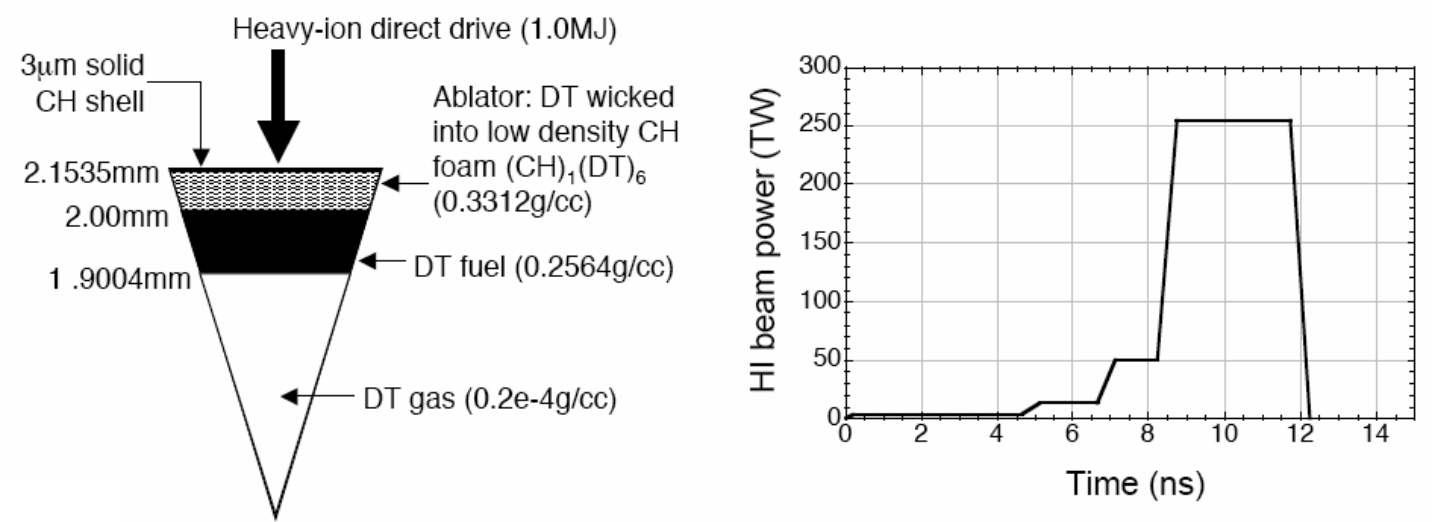

FIG. 2. A "pie" diagram of the target construction for the LASNEX calculation (2a), and the $50 \mathrm{MeV}$ Argon ion beam power pulse shape used (2b). This case gives a fusion gain of 50 for $1 \mathrm{MJ}$ of beam drive, assuming the incident beam profile zooms in a factor of two in radius (from $2 \mathrm{~mm}$ to $1 \mathrm{~mm}$ ) during the drive.

Table I shows beam and ablator plasma parameters at the four indicated times during the implosion. Note the large increases in ion range at constant incident Argon ion energy of $50 \mathrm{MeV}$, as the plasma temperature rises with the rising beam power (Fig.2) during the drive pulse. The electron drag decreases as the electron thermal speed exceed the ion beam speed above $\mathrm{Te}=460 \mathrm{eV}$. This allows the beam penetration (ends of the ion range)

Table I Beam and target ablator plasma values at four times during the implosion calculation of FIG. 2.

\begin{tabular}{|c|c|c|c|c|c|c|}
\hline $\begin{array}{c}\text { Implosion } \\
\text { Stage time (ns) }\end{array}$ & $\begin{array}{c}\text { Radii of peak } \\
\text { drive pressure } \\
\text { (cm) }\end{array}$ & $\begin{array}{l}\text { Radii of the } \\
\text { ends of beam } \\
\text { range }(\mathrm{cm})\end{array}$ & $\begin{array}{c}\text { Rho-r beam } \\
\text { ranges } \\
\left(\mathrm{mg} / \mathrm{cm}^{2}\right)\end{array}$ & $\begin{array}{c}\text { Plasma density } \\
\text { @ } 50 \% \text { beam } \\
\text { deposition }\left(\mathrm{g} / \mathrm{cm}^{3}\right)\end{array}$ & $\begin{array}{c}\text { Plasma temperature } \\
\text { a 50\% beam } \\
\text { deposition }(\mathrm{eV})\end{array}$ & $\begin{array}{c}\text { X-ray energy } \\
\text { into } 2 \pi \text { rẹl tọ } \\
\text { HI beaṃ } \\
\text { poweṛ } \\
\text { absorbed } \\
(\mathrm{kJ} / \mathrm{kJ})\end{array}$ \\
\hline $\begin{array}{c}\text { Halfway } \\
\text { through foot } \\
\text { pulse } 2.45\end{array}$ & 0.206 & 0.207 & 1.86 & 0.11 & 18 & $0.004 / 3.67$ \\
\hline $\begin{array}{l}\text { Halfway } \\
\text { through power } \\
\text { rise } 7.7\end{array}$ & 0.1940 & 0.1942 & 2.07 & 0.019 & 419 & $0.55 / 72.4$ \\
\hline $\begin{array}{c}\text { Halfway } \\
\text { through main } \\
\text { pulse } 10.25\end{array}$ & 0.1656 & 0.1650 & 4.28 & 0.037 & 1024 & $32 / 556$ \\
\hline $\begin{array}{c}\text { End of beam } \\
\text { drive } 12,0\end{array}$ & 0.1036 & 0.1037 & 6.31 & 0.027 & 1460 & $49 / 1000$ \\
\hline
\end{tabular}

to closely follow the imploding ablation front, as indicated by comparing the columns labeled "Radii of peak drive pressure", and "Radii of the ends of the beam range" in Table I. Because of the steep density and temperature gradients behind the ablation front, we find that electron thermal heat conduction is sufficient to transport the final 
third of the ion beam energy deposition during the peak ion beam power. However, comparing the last column of Table 1 with the input beam power shown in FIG. 2, we conclude that X-ray drive is not a dominant contribution to ablation drive pressure in this regime.

Figure $3 \mathrm{a}, \mathrm{b}$, c plots the results of integrating Eq. (1) for the Argon ion energy, beginning at $50 \mathrm{MeV}$ at $\rho \mathrm{r}=0$, as a function of beam penetration in units of $\rho r\left(\mathrm{~g} / \mathrm{cm}^{2}\right)$, at the first three implosion times in Table 1. (Plotting the same curves as a function of radius would make it difficult to distinguish the curves of deposition because of density gradients near the ablation front). Figure 3 curves $\mathrm{a}, \mathrm{b}$ and $\mathrm{c}$ show significant range-lengthening due to $\mathrm{v}_{\mathrm{b}}<\mathrm{v}_{\mathrm{eth}}$, especially near the ends of the ion ranges.

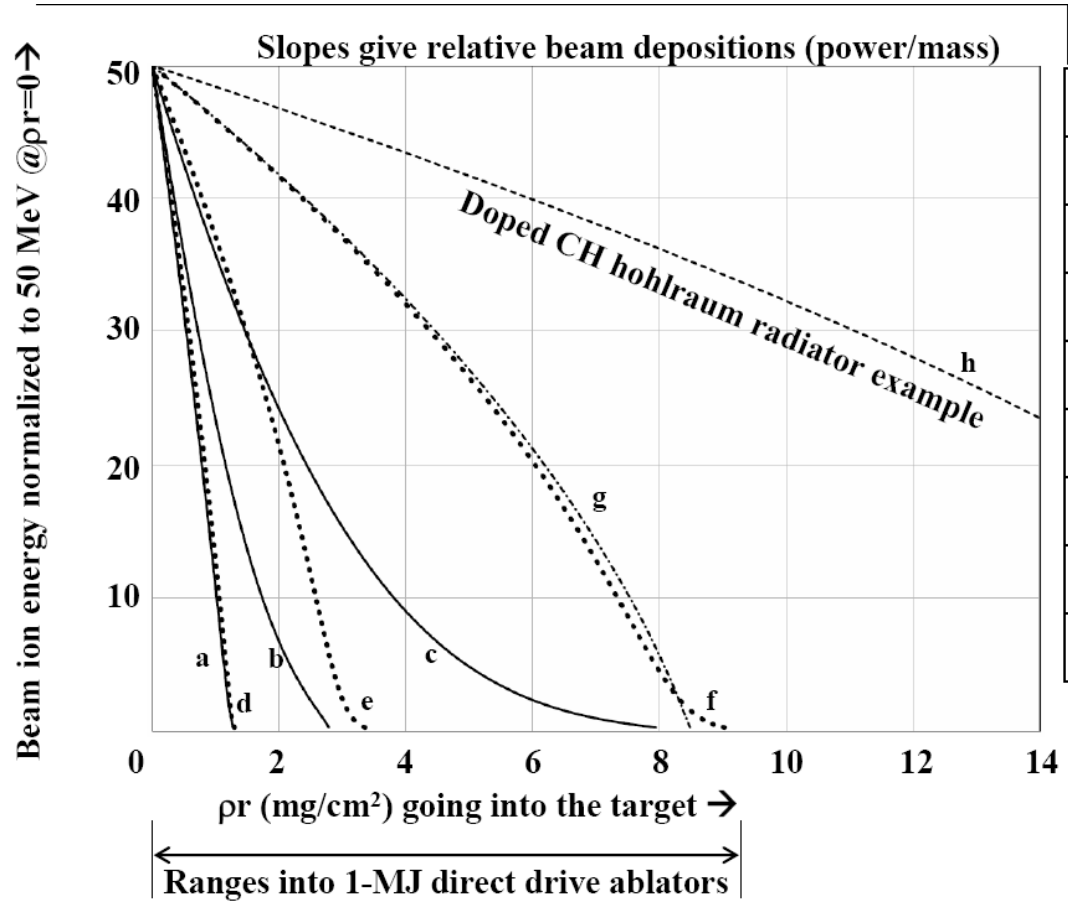

\begin{tabular}{|c|c|c|c|}
\hline \multicolumn{4}{|c|}{ Legend-all Argon beams } \\
\hline Line & Mat. & $\mathbf{E}_{\text {beam }}$ & $\mathbf{T}_{\mathrm{e}}$ \\
\hline $\mathbf{a}$ & CDT & 50 & 20 \\
\hline b & CDT & 50 & 400 \\
\hline c & CDT & 50 & 1000 \\
\hline ......... & H & 100 & 20 \\
\hline e & H & 200 & 200 \\
\hline f & H & 400 & 400 \\
\hline $\mathbf{g}$ & $\mathbf{H}$ & 400 & 20 \\
\hline h & $\mathrm{CH}$ & 400 & 200 \\
\hline & & $(\mathrm{Me}$ & $(\mathrm{eV})$ \\
\hline
\end{tabular}

FIG. 3. Argon beam ion energy as a function of penetration $\rho$ r into the ablated plasma/absorber, for the first three implosion times given in Table 1, for various ablator materials at equal mass (except for (h), incident ion energies, and electron temperatures.

The next four curves d, e, f and $g$ in Fig. 3 are predictions of the same dE/dx model if the ablator were pure hydrogen instead of the CH:DT ablator of Fig.2, and shows that with such a substitution, for the same ablator mass, the ion kinetic energies during the implosion would need to increase strongly in time to provide nearly the same beam penetrations in $\rho$ r vs. time, requiring a ramped beam voltage analogous to kind of accelerators considered in 
ref. [12]. The last curve in Fig. 3, (h), shows that much more mass is required for a $\mathrm{CH}$ ablator/absorber to stop the same ion energy, and that is why the estimated drive power is significantly higher for the equivalent size spherical hohlraum (Fig.1b). The strongly increasing ion range during the drive pulse is the key reason the constant $50 \mathrm{MeV}$ ion energy run resulted in a high overall coupling efficiency of $16 \%$ (1 MJ of beam input energy giving $150 \mathrm{~kJ}$ of fuel stagnation energy for ignition), and that future studies with higher ion energies and increasing in time should also enable high coupling efficiencies for the same reason. In fact, increasing the ion energy generally is helpful to reducing beam currents for the same power, and thus mitigating space charge problems in the accelerator drivers, and in addition, ramping the ion energy in time is useful for longitudinal beam compression (beam power amplification) to drive targets.

In Fig. 3 we normalize the other derived ion argon energies (where higher than $50 \mathrm{MeV}$ ) to plot on the same scale of 1 to 50 at $\rho r=0$ so that for the same incident beam power, the curves can be compared for relative local beam energy deposition power density (power per unit mass) vs. $\rho$ r and time. Thus with $\mathrm{H}$ ablators, which have more stopping power than DT ( $\mathrm{H}$ has 2.5 times as many electrons per unit ablator mass compared to DT), the ion speeds required for equal penetration vs. time are faster than $\mathrm{v}_{\mathrm{e}}$. As a result of the so-called "Chandrasekhar factor" $\mathrm{G}(\mathrm{x})$ dependence of $\mathrm{dE} / \mathrm{dx}$ (Eq. 1.), ion ranges with $\mathrm{H}$ ablators thus become insensitive to plasma electron temperature, seen by comparing curves $g$ and $f$ at the same beam energy but with different ablator temperatures. In the regime where $\mathrm{v}_{\mathrm{b}}>\mathrm{V}_{\mathrm{eth}}$, R. Piriz has developed a useful analytic model [15] for predicting the hydrogen ablation profiles of velocity, temperature, density, etc. Note in Fig. 3 d, e, and f that for the higher ion energies planned using $\mathrm{H}$ ablators, the slopes in the last third of the deposition ranges (the portions that are most important to driving the ablation front pressure), are almost twice has high as for the $50 \mathrm{MeV}$ argon deposition curves a, b, c, while the slopes over the first third of ion deposition, representing parasitic ion losses on outgoing ablated plasma, are half of what they are for the $50 \mathrm{MeV}$ beam cases after the initial foot. Setting RT-stability considerations aside, we expect much higher coupling efficiencies with $\mathrm{H}$ ablators compared to the CDT ablators used in the LASNEX runs (Fig. 2), but we also expect more RT instability without using some mitigating measures (see Sec. III).

Figure 4 shows target gain (4a) and overall coupling efficiency (4b) for fuel capsules similar to Fig. 2 but with various ablator thicknesses ( $\rho r)$ for two different ablator materials-the ablator indicated in Fig.2-CH foam wicked with DT, labeled DT/CH, and for a pure DT ablator. The maximum coupling efficiency we found for a pure 
DT ablator has a lower target gain because of preheat (pure DT has very low opacity even for very soft x-rays). Less than $1 \%$ carbon doping in a hydrogen ablator is estimated to be needed to prevent this preheat in future calculations.
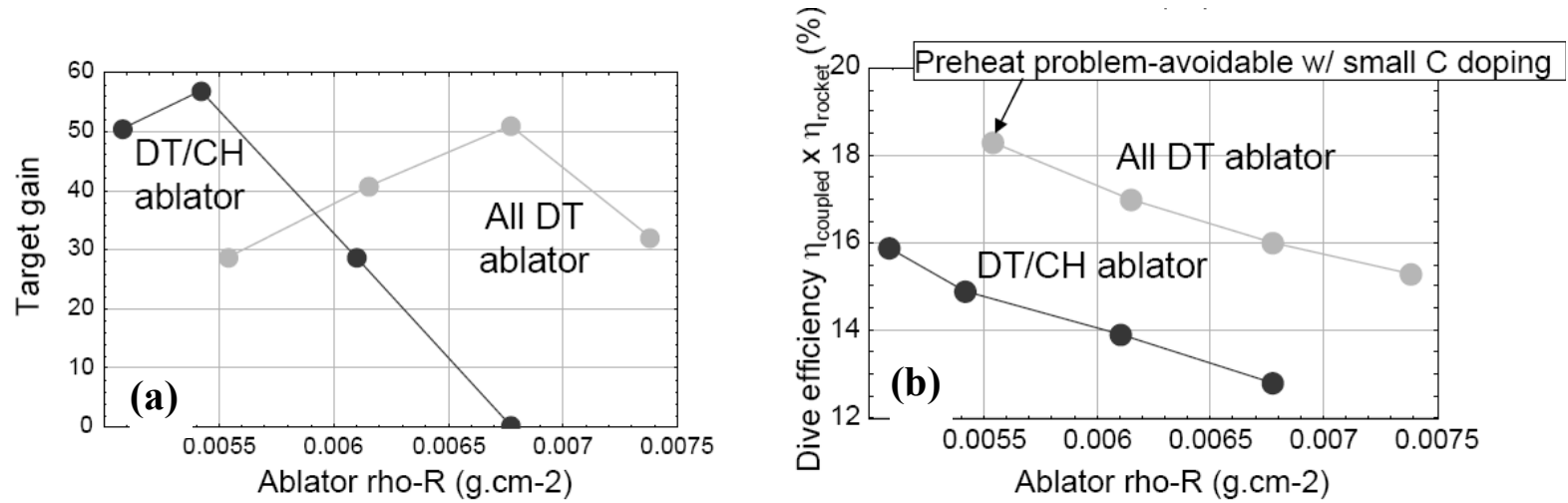

FIG. 4. LASNEX 1-D results for target gain (a) and drive coupling efficiency (b) as a function of ablator $\rho r$ $\left(\mathrm{g} / \mathrm{cm}^{2}\right)$ for the same fuel capsule and incident beam energy as in Fig. 2, (but retuning the pulse shape).

\section{Section III. Summary and Discussion of Future Work Needed}

Our LASNEX results for heavy ion beam to target coupling efficiency in direct drive $16 \%$ with CH:DT ablator, and $18 \%$ for pure DT ablator, are the highest overall coupling efficiencies for published 1-D implosion calculations, to our knowledge, for ignition with adequate gain for efficient accelerators, at a total beam input energy of $1 \mathrm{MJ}$. We have not yet optimized these 1-D results, and we may expect higher coupling efficiencies in future work using ramped ion beam energies to get ramped ion ranges with high stopping-power hydrogen ablators, because of the more favorable deposition profiles noted in Figure 3. We also note that the implosion velocity of $4.45 \times 10^{7} \mathrm{~cm} / \mathrm{s}$ is much higher than the minimum needed for ignition. The National Ignition Facility, for example, is designed to test ignition with $0.24 \mathrm{mg}$ of DT fuel ( $0.45 \mathrm{mg}$ including residual Beryllium ablator) at $3.68 \times 10^{7} \mathrm{~cm} / \mathrm{s}$ peak implosion velocity, a total fuel payload energy, including the residual Beryllium pusher, of $30 \mathrm{~kJ}$, producing $20 \mathrm{MJ}$ of fusion yield [16]. This NIF capsule design absorbs $200 \mathrm{~kJ}$ of hohlraum x-rays, for a "capsule" coupling efficiency of $15 \%$, about the same efficiency as we calculate here for ion direct drive with low Z CH:DT ablators. In future work, we will extend this work to consider driving low-Z capsules the size of the NIF capsule with heavy ion beams (assuming we can focus heavy ion beams to the $1 \mathrm{~mm}$ radius target size, with say, heavier Krypton ions for the lower ranges required, and with short-focal-length copper final-focus magnets). If successful designs emerge, and, if the NIF's ignition campaign is also successful, the prospects for heavy ion fusion development might look much different: gain 100 at $200 \mathrm{~kJ}$ total drive energy! Further into the future, if we can optimize coupling efficiencies to, 
say, $25 \%$ for larger mass targets, then large fuel assembly energies of $1 \mathrm{MJ}$ might be possible with $4 \mathrm{MJ}$ of beam drive energy. Such large fuel assembles with T-lean fuel $[17,18]$ (DD fuel with a small inner DT sparkplug) at compressed $\rho r \sim 10 \mathrm{~g} / \mathrm{cm}^{2}$ would self-breed tritium without external blankets, and internally capture the neutron energy into mostly plasma energy for direct conversion. In that event, prospects for fusion energy might be radically changed also.

As we noted previously, beam deposition profiles which enhance coupling efficiency may also result in increased Rayleigh Taylor instability growth rates, and so final conclusions for maximum coupling efficiency cannot be drawn until constraints set by RT instability are examined, which will require 2-D and maybe 3-D implosion calculations. In general, concentrating beam energy deposition as close as possible behind the ablation front and following it in time with increasing ion range maximizes coupling efficiency, but may also generate higher local pressure gradients and faster classical RT growth rates. Just to illustrate how important RT instability could be, we can make a rough estimate of the maximum growth rate (neglecting any ablative stabilization)

$$
\gamma=(A g k)^{1 / 2}
$$

where the Atwood number $\mathrm{A}=\left(\rho_{1}-\rho_{2}\right) /\left(\rho_{1}+\rho_{2}\right), \mathrm{g}$ is the peak acceleration, and $\mathrm{k}$ the wave number, typically taken to be $2 \pi /$ remaining ablator thickness. Estimating peak pressure gradients near the ablation front from parameters in Table 1 using $\mathrm{A} \sim 0.5$ for H/DT, we derive an acceleration $\mathrm{g} \sim \mathrm{v}_{\text {imp }} / \tau_{\text {peak }} \sim 10^{16} \mathrm{~cm} / \mathrm{s}^{2}$, and $\mathrm{k} \sim 6 \times 10^{2} \mathrm{~cm}^{-1}$ yielding $\gamma$ $\sim 1.7 \times 10^{9} \mathrm{~s}^{-1}$, or $\exp \left(\gamma \tau_{\text {peak }}\right) \sim 1000$, which is about the maximum tolerable growth factor for most high convergence ratio inertial fusion target designs. For the 1-D LASNEX run represented in Figs. 2 and 3, there are three factors that can mitigate RT growth: (1) in the most sensitive foot part of the drive, the ion beams penetrate only $25 \%$ of the ablator thickness, and the density of the mostly DT ablator is close to that of the DT fuel payload (a small effective Atwood number); (2) the ion beam deposition power density falls off towards the end of the ion range (Fig. 3 a, b, c curves) thus reducing the pressure gradients behind the ablation front; this also reduces the coupling efficiency, which is still high nonetheless (16\%); (3) heavy-ion direct drive may exhibit appreciable ablative stabilization of higher mode number RT growth as discussed above. The shape of those deposition profiles look like the more stable ones with ion energy spread in ref. 9. On the other hand, the curves Fig. d, e, f, for higher energy ramped ion beams can be expected to have higher coupling efficiency but also can be expected to be more unstable. We conclude from this discussion that constraints on ion direct drive coupling efficiency need to be assessed with 2-D calculations including RT growth from seeded perturbations with a spectrum of wave numbers. 
For fixed radial pointing, the number of beams is considered a major factor determining beam non-uniformity seeding RT growth [19, 20, 8]. However, noting the beneficial effect of off-radial ion trajectories and beam energy spread in [9] for reduced RT growth rates, we suggest delivering most ion beam energy in off-radial trajectories, which we call oblique irradiation, by use of hollow beam spot profiles as depicted in Figure 5, created by rapid beam spot rotation. We expect the same multi-GHz RF modulation of ion beams as proposed by Sharkov [21] for driving cylindrical heavy ion targets with a hollow, rotating beam spot can also be applied to provide a hollow beam for heavy ion direct drive of spherical ablators with mostly oblique incident ion rays. A series of phased RF cavities would be used to impress a helical beam centroid variation upstream of the target before beam drift compression and focusing; this perturbation maps into hollow beam spots on the target with radii controlled by the RF amplitude.

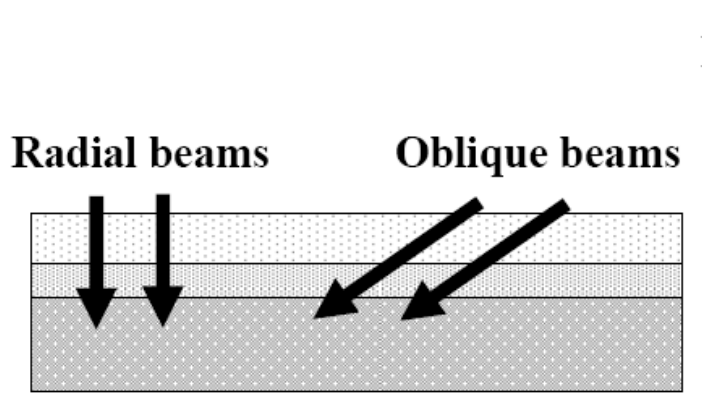

(a) Un-perturbed ablator

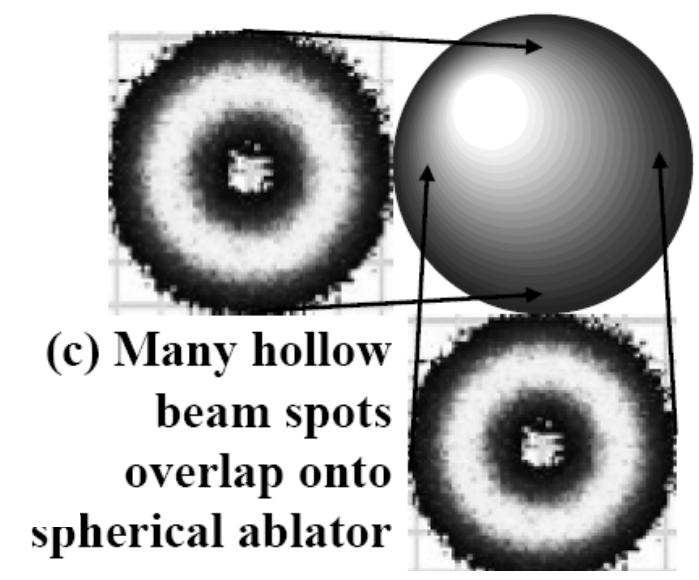

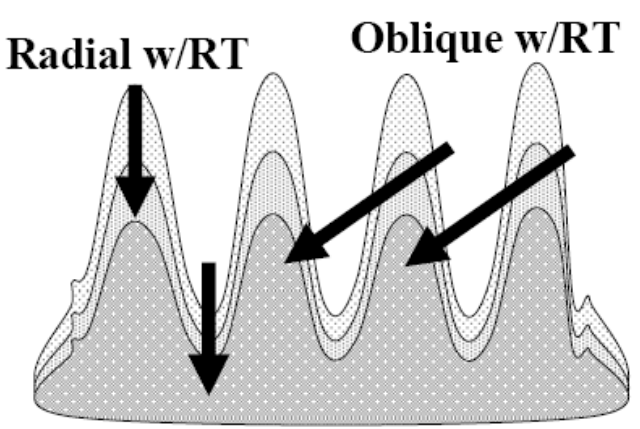

(b) Perturbed ablator.
|| Acceleration

(g)

Density gradient

\section{(d) RF wobbler to create hollow, rotated beam spots (Sharkov)}

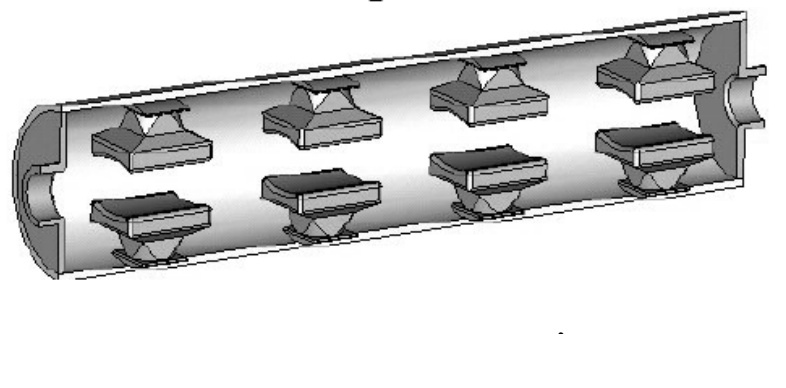

FIG. 5. Comparison of oblique as opposed to radial ion beam deposition using rotated beam spots onto direct drive target ablators (proposed). (a) un perturbed ablator; (b) perturbed ablator, (c) projection of hollow beam spots onto a spherical ablator, for smoothing and mitigation of RT instability; (d) schematic of a series of phased R. RF cavities that can produce and control hollow beams (after Sharkov [21]). 
The RF amplitude can be reduced in time (amplitude decreasing during the beam pulse) such that the radius of the hollow beam projected onto the target ablator surface would shrink during the implosion (zooming). Rotating beams may provide smoother beam deposition uniformity with fewer beams. In addition, S. Kawata has suggested that the pulsating nature of the beam energy deposition with a rotating beam spot may produce a type of dynamic stabilization [22].

Finally, we point out that determination of the number of beams with time-dependent beam energy and irradiation geometries that can provide sufficient uniformity and stability for ion direct drive using 2-D calculations are pre-requisite to designing compatible target chambers/final focus magnets, and any new target fabrication and injection methods to be developed.

Acknowledgements: This work has benefited from many helpful discussions with Roger Bangerter, David Bailey, Alex Friedman, Max Tabak, Boris Sharkov, and Shigeo Kawata. This work was performed under the auspices of the US Department of Energy by Lawrence Livermore National Laboratory under contract DE-AC52-07NA27344 and the University of California, Lawrence Berkeley National Laboratory under contract DE-AC02-05CH1123.

\section{References}

[1] J. Nuckolls, L. Wood, A. Thiessen, and G. Zimmerman, Nature 239, 139 (1972)

[2] J. D. Lindl, Inertial Confinement Fusion: the Quest for Ignition and Energy Gain Using Indirect Drive (AIP Press, New York, 1998).

[3] G. Allshouse and D. Callahan, Nuc. Fusion 39, No. 71999

[4] D. Callahan and M. Tabak, Phys. of Plasmas 7, No. 5, May 2000

[5] N. Metzler and J. Meyer-Ter-Vehn Laser Part Beams 2, part 1, p27-49 (1984).

[6] K. A. Long and N. A. Tahir, Phys. Rev. A 35, No. 6, March 1987

[7] T. Someya, K. Miyazawa, T. Kikuchi, and S. Kawata, Laser Part. Beam 24, 359 (2006)

[8] S. Atzeni and J. Meyer-Ter-Vehn, The Physics of Inertial Fusion, (Clarendon Press, Oxford, 2004) p.233

[9] R. O. Bangerter and D. J. Meeker "Charged Particle Fusion Targets", in proceedings of the 2nd International Topical Conference on High Power Electron and Ion Beam Research, Ithaca, New York, October 3-5, 1977 Lawrence Livermore National Laboratory report UCRL-79875 Nov. 9, 1977. Available through NTIS: NTISUCRL79875. 
[10] G. B. Zimmerman, W. L. Kruer W.L, Comments on Plasma Phys. Controlled Fusion 251 (1975). Most recent updates were provided by T. Kaiser, G. Kerbel, and M. Prasad, private communication, (2005).

[11] G. R. Magelssen, Nuclear Fusion 24, No.12, p. 1527, 1984D.

[12] Havazelet, M. Sapir, and T. Bar-Noy, J. Phys. D: Appl. Phys., 16, p. 315-319 (1983).

[13] P. K. Roy, S. S. Yu, E Henestroza, A Anders, F M Bieniosek, J Coleman, S Eylon, W G Greenway, M Leitner, B G Logan, W L Waldron, D R Welch, C Thoma, A B Sefkow, E P Gilson, P C Efthimion, and R C Davidson Phys. Rev. Lett. 95, 234801(2005)

[14] T. Kaiser. G. Kerbel, and M. Prasad, unpublished LLNL presentation "Implementing Ion Beams in Kull and Hydra [1999]; see also, e.g., S. Atzeni and J. Meyer-Ter-Vehn, The Physics of Inertial Fusion, (Clarendon Press, Oxford, 2004) p.389.

[15] A. R. Piriz, Plas. Phys. and Cont. Fus. 28, No 8, p 1167 (1986)

[16] J. D. Lindl, Phys Plasmas 11339 (2004)

[17] M. Tabak, Nuclear Fusion 36, No 2 (1996)

[18] S. Atzeni and C. Ciampi, Nuclear Fusion 37, 1665 (1997)

[19] R. Sacks, R. Arnold, and G. Magelssen, Nuc. Fusion 221421 (1982)

[20] S. Kawata and K. Niu J. Phys. Soc. Japan 53, 3416 (1984)

[21] B. Sharkov, in proceedings of the Inertial Fusion Science and Applications Conference, Kobe, Japan 2007 plenary paper "Heavy Ion Fusion Drivers", to be published in Journal of Physics: Conference Series

[22] S. Kawata has suggested that non-uniform and time varying accelerations such as induced by beam spot rotation and multi-pulsing in time may reduce the RT growth rates-see linear RT growth analysis in Kawata, et al, Laser and Part. Beams 11, No.4 (1993). 